\title{
Airway immunometabolites fuel Pseudomonas aeruginosa infection
}

\author{
Sebastián A. Riquelme and Alice Prince*
}

\begin{abstract}
Pulmonary infections are associated with a brisk inflammatory reaction to bacterial surface components. Lipopolysaccharides (LPS) trigger macrophage activation and release of mitochondrial metabolites that control the intensity of the immune response. Whereas succinate induces oxidative stress (ROS), HIF1a stabilization, glycolysis and IL-1 $\beta$ release, itaconate suppresses inflammation by inhibiting succinate oxidation, glycolytic flux and promoting antioxidant Nrf2-HO-1 functions. P. aeruginosa is a major pathogen associated with acute and chronic lung infection. Although both secreted toxins, LPS and proteases are key factors to establish acute $P$. aeruginosa pneumonia, lack of these components in chronic P. aeruginosa isolates suggest these organisms exploit other mechanisms to adapt and persist in the lung. Upon inhalation, P. aeruginosa strains trigger airway macrophage reprograming and bacterial variants obtained from acutely and chronically infected subjects exhibit metabolic adaptation consistent with succinate and itaconate assimilation; namely, high expression of extracellular polysaccharides (EPS), reduced IptD-LPS function, increased glyoxylate shunt (GS) activity and substantial biofilm production. In this review we discuss recent findings illustrating how P. aeruginosa induces and adapts to macrophage metabolites in the human lung, and that catabolism of succinate and itaconate contribute to their formidable abilities to tolerate oxidative stress, phagocytosis and immune clearance.
\end{abstract}

Keywords: Pseudomonas aeruginosa, Pneumonia, Succinate, Itaconate, Immunometabolism, Biofilm, Adaptation, Cystic fibrosis, ROS, Metabolic stress

\section{Background}

Opportunistic bacterial pathogens, such as Pseudomonas aeruginosa, Klebsiella pneumoniae and Staphylococcus aureus are frequently associated with persistent pulmonary infection $[1,2]$. These pathogens are a major cause of morbidity and mortality, especially in individuals with damaged airways, as occurs in ventilator associated pneumonia (VAP) [3-6], in subjects with antecedent viral infection [7-10], or in patients exhibiting airway inflammation, as in chronic obstructive pulmonary disease (COPD) [11, 12] and in cystic fibrosis (CF) [1316]. Antibiotic resistance is a common feature of these organisms, and may contribute to intractable infection, but even susceptible strains are able to cause chronic

*Correspondence: asp7@columbia.edu

Department of Pediatrics, Columbia University, New York, NY 10032, USA inflammation and eventual mortality, suggesting mechanisms other than drug resistance are involved in pulmonary pathogenesis. It is also curious that ex vivo, many of these bacteria are readily phagocytosed and killed by immune cells, suggesting that conditions within the airway itself, such as the complex metabolic milieu provided by inflammatory cells, may contribute to bacterial survival $[1,2]$. The ability of these major opportunists to form biofilms, which protect bacteria from antibodies, complement, phagocytosis, antibiotic penetrance and especially from oxidants is a common factor in their pathogenicity and clearly contributes to their shared ability to cause persistent pulmonary infection $[11,17,18]$. Exactly what signals from the host activate the formation of biofilm are not well defined.

Biofilm formation results when a community of bacteria are able to form a nidus of infection on a surface, original author(s) and the source, provide a link to the Creative Commons licence, and indicate if changes were made. The images or other third party material in this article are included in the article's Creative Commons licence, unless indicated otherwise in a credit line to the material. If material is not included in the article's Creative Commons licence and your intended use is not permitted by statutory regulation or exceeds the permitted use, you will need to obtain permission directly from the copyright holder. To view a copy of this licence, visit http://creativecommons.org/licenses/by/4.0/. The Creative Commons Public Domain Dedication waiver (http://creativeco mmons.org/publicdomain/zero/1.0/) applies to the data made available in this article, unless otherwise stated in a credit line to the data. 
from an initial inoculum of planktonic organisms [19]. Biofilm-forming organisms attach to mucosal surfaces or form aggregates that are almost impossible for myeloid cells to engulf. This lifestyle is a defense response to oxidant stress [20] and can be triggered in vitro by reactive oxidant species (ROS) such as hydrogen peroxide $\left(\mathrm{H}_{2} \mathrm{O}_{2}\right)$ $[21,22]$. Biofilms generate a multilayered shield of extracellular polysaccharides (EPS), which are produced in response to both metabolic stress and structural damage to the bacteria. EPS functions as an oxidant sink [20, $23,24]$, and the bacterial pathways that generate EPS components function to dissipate oxidant stress either by producing ROS-scavenging byproducts or by simply decreasing the metabolic rate to decrease endogenous ROS synthesis $[25,26]$. The pathogenesis of $P$. aeruginosa infection in patients with CF provides an especially well-studied example of the development of pulmonary infection and biofilm formation in vivo in response to the pro-oxidant environment $[15,16]$. While there have been numerous theories to explain the specificity of $P$. aeruginosa for the CF airways, there are ample data demonstrating that the infecting organisms form biofilms in vivo and that these bacterial communities are a major factor in the limitations of antimicrobial therapy in this disease [15, 27-29]. A prominent characteristic of the CF airway is the substantial accumulation of immune cells, phagocytes and $\mathrm{T}$ cells, which generate oxidant and inflammatory mediators that damage the lung [14]. These cells produce metabolites that determine the airway oxidant profile in response to $P$. aeruginosa. Such conditions select for bacteria with the metabolic plasticity that enables bacterial exploitation of these immune byproducts for the production of biofilms $[2,30]$.

$\mathrm{CF}$ is a genetic disease caused by mutations in the $\mathrm{CF}$ transmembrane conductance regulator (CFTR) that results in an altered accumulation of airway metabolites and ions, especially succinate, itaconate, chloride and bicarbonate $[30,31]$. As these patients are typically followed from infancy, often before the establishment of pulmonary infection, through adulthood, they provide the unique opportunity to follow the adaptation of bacteria to the human airway over prolonged periods of time. This provides the opportunity to see how bacteria alter their own gene expression once they take up residence in the lung. The adaptation of $P$. aeruginosa to the CF lung provides insights into the host factors that initiate and promote chronic infection, as well as how tightly controlled the metabolic immune response must be to avoid excessive oxidant stress and P. aeruginosa biofilm development. In this review, we will explore how the accumulation of the immunometabolites succinate and itaconate provides a milieu that initially promotes the proliferation of $P$. aeruginosa, then generates selection for variants that produce abundant EPS instead of LPS, generates intractable biofilms and enables chronic infection.

\section{Main text \\ Macrophage activation and inflammation in the infected airway}

The human airway is replete with carbon sources for bacterial proliferation, essential microelements such as iron $\left(\mathrm{Fe}^{++}\right)$and nutrients like amino acids and lipids [32]. Ubiquitous opportunists, such as $P$. aeruginosa commonly found in the environment, colonizing sinks and showers as well as streams, are often inadvertently inhaled. The vast majority of these organisms are rapidly cleared, but those that persist must forcibly adapt to the microenvironment provided by the airway.

Much of what is known about the composition of airway fluid has been obtained from studies of CF patients, focusing primarily upon the concentrations of $\mathrm{Na}^{+}, \mathrm{Cl}^{-}$, $\mathrm{HCO}^{-}$and water in the setting of infection [32-34]. In studies using LPS as a surrogate for bacterial infection, macrophages very rapidly switch their resting metabolic activity from oxidative phosphorylation (OXPHOS) to glycolysis, with the resulting accumulation, oxidation and release of succinate and its byproduct ROS [35-38]. Both metabolites activate the hypoxia induce factor $1 \alpha$ $(\mathrm{HIF}-1 \alpha)$ and IL-1 $\beta$ production initiating a proinflammatory response [35-37] (Fig. 1a, b), which is associated with the recruitment and activation of phagocytes. Thus, IL- $1 \beta$ is an important proinflammatory cytokine expected to facilitate the eradication of $P$. aeruginosa. However, in several models of pneumonia, IL-1 $\beta$, in fact, contributes to $P$. aeruginosa infection [39-41]. Mice lacking a receptor for IL-1 $\beta\left(i l 1 r^{-/}\right)$or those treated with its antagonist Anakinra actually clear $P$. aeruginosa significantly better than those with a robust IL-1 $\beta$ response. The inflamed CF airway with many activated macrophages and monocytes is enriched for succinate, in comparison to normal control broncheoalveolar lavage (BAL) fluid, and elevated amounts of succinate have also been observed in animal models mimicking the CF disease [30, 42, 43] (Fig. 1a, b). Thus, an important substrate for bacterial proliferation, succinate, is immediately available for bacterial consumption upon their entry into the airway, and is provided by activated myeloid cells.

\section{Succinate is a preferred carbon source for environmental $P$. aeruginosa}

Many different types of inhaled bacteria are likely to be entrapped by the dehydrated airway secretions that are prominent in CF; but in fact, only a few species cause persistent infection. These opportunists, most prominently $P$. aeruginosa must rapidly adapt to the available metabolites, including macrophage succinate. In contrast 

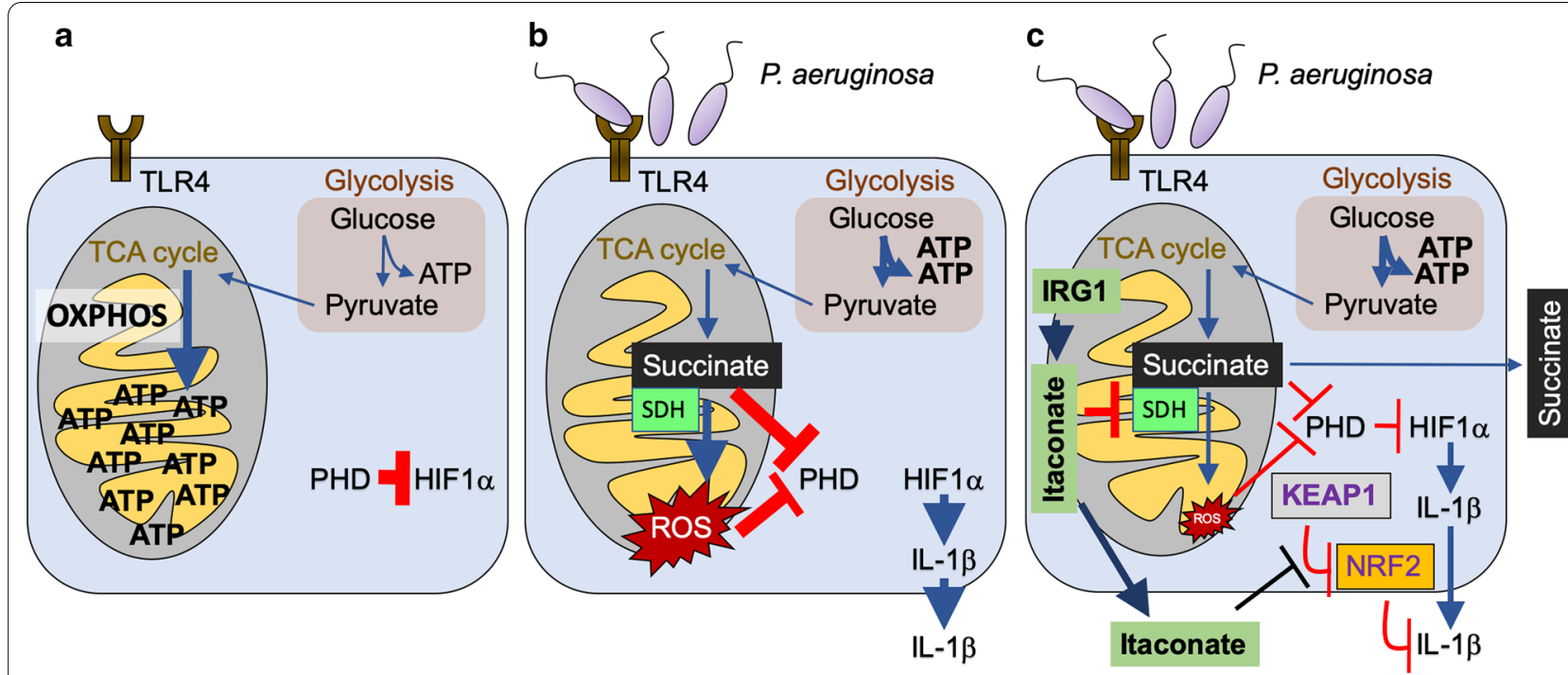

Fig. 1 Immunometabolites succinate and itaconate are released during airway macrophage infection. a During homeostasis, airway macrophages employ the TCA cycle and OXPHOS to generate energy (ATP) in the mitochondria. In these conditions, the pro-inflammatory transcription factor HIF1a is inhibited in the cytoplasm by prolylhydroxylases (PHD). b Upon bacterial LPS detection by toll-like receptor 4 (TLR4), cells exhibit metabolic reprograming. Mitochondria become a major source for reactive oxygen species (ROS), which is mostly derived from succinate accumulation and its oxidation by succinate dehydrogenase (SDH). Succinate and ROS inhibits PHD, which release HIF1a to promote transcription of pro-inflammatory cytokines like IL-1 $\beta$. Glycolysis becomes the major ATP source. c To avoid excessive tissue oxidation, macrophages also upregulate Immunoresponsive Gene 1 (IRG1), which synthetizes the SDH and KEAP1 inhibitor itaconate. SDH blockade induces succinate accumulation, which is excreted from the cell together with itaconate. Reduced SDH function by IRG1 regulates airway HIF1a and IL-1 $\beta$ activity. Itaconate is bactericidal

to many other bacteria that prefer glucose or amino acids, $P$. aeruginosa preferentially consumes succinate as directed by its $\mathrm{crc}$ locus, before utilizing other carbon sources [44-48] (Fig. 2a). However, forced succinate consumption generates substantial oxidant stress for the organisms, which are already in an oxidant rich environment with activated phagocytes releasing ROS [14, 20, $24,48]$. As a response, $P$. aeruginosa variants are selected that utilize the anti-oxidant glyoxylate shunt (GS) to dissipate ROS and to generate biofilm, which itself has antioxidant properties [25, 26, 30, 49].

Laboratory strains of $P$. aeruginosa grown in high levels of succinate demonstrate metabolic changes that enable them to proliferate amidst high levels of oxidants [30] (Fig. 2b). These bacteria induce even more succinate in the airway as well as myeloid cell death, consistent with increased secretion of IL- $1 \beta$ and pyroptosis [30]. Succinate-exposed strains increased their glucose metabolism and utilization of threalose and acetate, which feed the GS, production of EPS and enable tolerance to oxidant stress. P. aeruginosa grown in high succinate were phenotypically different, with increased colony size, consistent with the abundant production of EPS. These strains caused significantly greater levels of infection in mouse models of pneumonia, decreased myeloid cell viability and promoted more IL- $1 \beta$ release. Importantly, the same constellation of metabolic and anti-oxidant changes was identified in a collection of clinical $P$. aeruginosa strains cultured from an adult with CF [30, 50, 51]; namely, altered carbon substrate utilization, increased use of the GS and expression of genes associated with EPS and biofilm formation. Both the clinical strains from CF and the $P$. aeruginosa grown in high succinate in vitro were more proficient in colonizing the airways of mice, causing persistent infection that lasted for days. Thus, the ability to metabolize succinate and modify their own metabolic activity in response to the ROS generated by this immunometabolite enables $P$. aeruginosa to proliferate and adapt to the pro-oxidant airway environment.

\section{Airway succinate is regulated by phosphatase and tensin homologue deleted on chromosome 10 (PTEN)}

PTEN, by controlling the P3K/Akt/mammalian target of rapamycin (mTOR) pathway regulates cellular proliferation, glycolytic metabolism [52] and mitochondrial activity [53-55]. PTEN participates in the regulation of mitochondrial respiration, especially controlling the function of cytochrome $\mathrm{C}[54]$ and the assimilation of the oxidative TCA cycle substrate isocitrate [30]. PTEN dysfunction induces increased expression of isocitrate dehydrogenase (IDH), which overstimulates mitochondrial complex I by providing with more nicotinamide adenine dinucleotide (NADH)[30]. Increased complex I activation induces more oxygen reduction and ROS, 

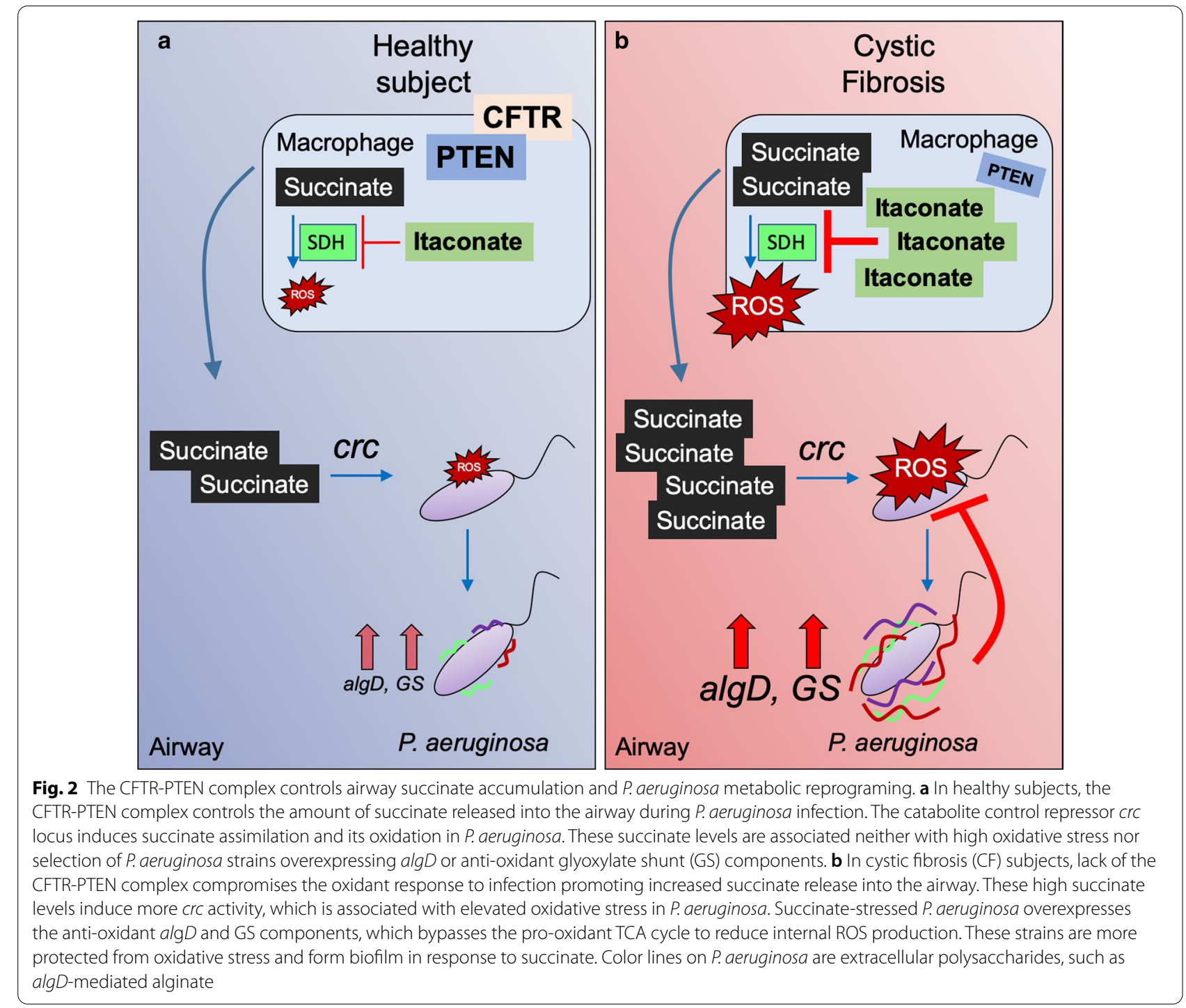

which, during LPS stimulation, is reinforced by electrons reversely transferred from complex II, succinate dehydrogenase (SDH)[37]. This excessive ROS production activates the anti-oxidant cell response, which, in part, is mediated by itaconate. This metabolite is the product of $\operatorname{Irg} 1$ (in humans Acod1), and its activity is linked to, for example, inhibition of SDH [56-59]. Thus, PTEN deregulation induces oxidative stress in mitochondria and, as a compensatory mechanism, itaconate synthesis, which inhibits SDH and enable succinate accumulation and its release [30,60]. For proper PTEN metabolic activity, this phosphatase associates with the C-terminal cytoplasmic tail of CFTR, which is known to increase its stability and activation by dephosphorylation [61]. In cells (and patients) with decreased membrane-associated CFTR, or in subjects harboring mutations in the CFTR C-terminal tail, there is also decreased PTEN numbers and increased succinate accumulation [42, 61] (Fig. 2). This deregulation is associated with more airway inflammation [61, 62]. $P$. aeruginosa infection of myeloid and epithelial cells induces both PTEN reduction and succinate release, and peripheral blood mononuclear cells (PBMCs) from individuals exhibiting CFTR-PTEN deficiency secrete much more succinate levels than controls after infection with these organisms [30,61]. The airway of CFTR-PTEN deficient subjects showed tenfold succinate accumulation respect with healthy individuals, demonstrating that the metabolic activation status of epithelial and myeloid cells can impact the pro-oxidant composition of the airway metabolome. As the CFTRPTEN association is not dependent upon the channel function of CFTR, therapeutic strategies that increase the membrane localization of CFTR should also 
increase PTEN and normalize succinate, inflammation and also reduce $P$. aeruginosa infection.

\section{Itaconate is an immunometabolite that protects the airway from oxidative damage}

Itaconate, an electrophilic carboxylate, is also a prominent metabolite found in the infected airway [30, 59] (Figs. 1c, 3). As described above, itaconate is released by LPS activated macrophages in response to succinate oxidation by SDH, and functions to counter the proinflammatory response caused by ROS and IL-1 $\beta$ [63]. Itaconate and its derivatives 4-octyl itaconate and dimethyl-itaconate suppress inflammation by targeting Kelch-like $\mathrm{ECH}$-associated protein 1 (KEAP1), which sequesters the anti-oxidant Nuclear Factor-Erythroid-2-Related Factor 2 (NRF2) transcription factor in the cytoplasm [64, 65]. Itaconate alkylates KEAP1, releasing NRF2 from proteosomal degradation and promoting its migration to the nucleus where it induces expression of multiple antioxidant genes such as HMOX1 (Heme-oxygenase 1), as well as suppress production of IFN $\beta$ [64]. Itaconate also suppresses secretion of inflammasome associated proinflammatory cytokines by dicarboxypropylation of pyrin domain-containing protein 3 (NLRP3), which reduces its interaction with NEK7 [66]. Reduced NLRP3-NEK7 complex formation abolishes the LPS-induced assembly of the inflammasome, preventing the production of, for example, IL-1 $\beta$, and in PBMCs from subjects exhibiting the IL-1 $\beta$ based disease cryopyrin-associated periodic syndrome (CAPS) itaconate and 4-octyl itaconate reduce IL-1 $\beta$ production.

During Gram-negative infection of mucosal surfaces, itaconate participates in the metabolic control of inflammation by suppressing release of pro-inflammatory cytokines by interrupting the oxidation of succinate by SDH $[63,67]$. Reduced transformation of succinate into fumarate diminishes the amounts of ROS required to inhibits prolyl hydroxylases (PHD), and impeding HIF1 $\alpha$ migration to the nucleus to promote transcription of proglycolytic and pro-inflammatory genes [35]. Reduced SDH function due to itaconate accumulation safeguards the integrity of the mucosal tissue, as it induces less local damage and accelerates the process of tissue repair. Itaconate also interacts with macrophage aldolase, where it induces cysteine modifications that reduce its catalytic function and, eventually, inactivating glycolytic flux and

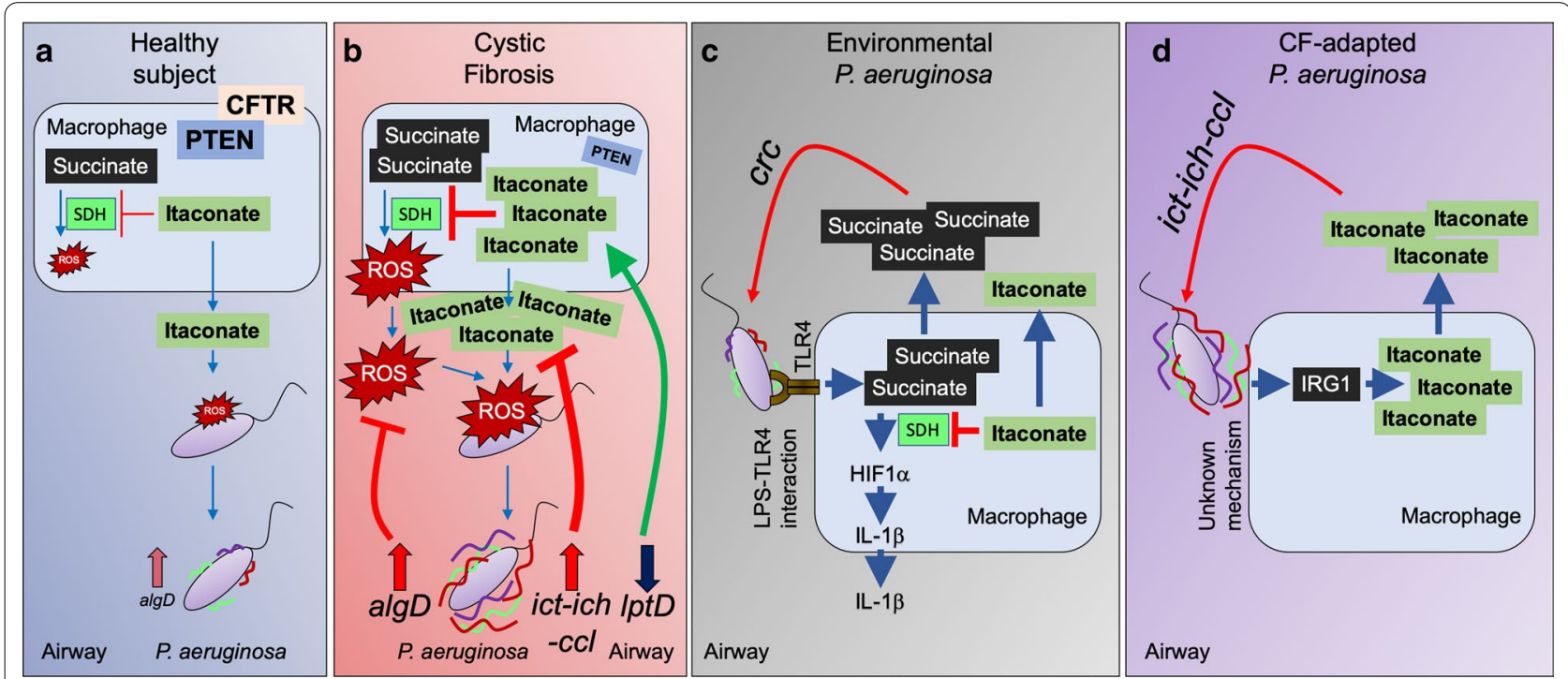

Fig. 3 Itaconate fuels $P$. aeruginosa adaptive changes and chronic infection. a In healthy subjects, itaconate is produced during $P$. aeruginosa infection to control SDH activity, oxidative stress and inflammation. This itaconate levels are tolerated by $P$. aeruginosa during acute infection. $\mathbf{b}$ In CF individuals lacking CFTR-PTEN complex activity, elevated succinate oxidation induces synthesis and release of the anti-oxidant molecule itaconate as a compensatory mechanism. Airway itaconate induces P. aeruginosa outer membrane stress, which induces ict-ich-ccl locus overexpression to degrade itaconate. Itaconate also induces downregulation of IptD, which suppresses surface exposure of LPS. Lack of surface-exposed endotoxin causes bacterial membrane deregulation and permeability, which is compensated by activation of the algT-algD membrane stress response to produce more protective alginate. Through an unknown mechanism, alginate induces more itaconate release by host macrophages, which fuels biofilm production, adaptation and long-term infection. $\mathbf{c}$ Environmental P. aeruginosa strains expressing LPS induce the TLR4-SUccinate-HIF1a-IL-1 $\beta$ axis, inducing release of succinate and regulatory itaconate. Succinate released fuels $P$. aeruginosa infection through the crc locus during acute infection. $\mathbf{d}$ Host-adapted $P$. aeruginosa isolates, which lack surface LPS and overproduce alginate, induce IRG1 expression and high itaconate production in macrophages. IRG1 induction is mediated by alginate. Itaconate released fuels host-adapted P. aeruginosa through the ict-ich-ccl locus activity. Color lines on P. aeruginosa are extracellular polysaccharides, such as algD-mediated alginate 
the release of effector cytokines that might harm the lung [68]. Irg1 null mice are highly susceptible to the hyperinflammatory pathology associated with LPS [63]. In other pulmonary infections, the lack of itaconate enables massive recruitment of myeloid cells to the Mycobacterium tuberculosis infected lung, exacerbating inflammation, disrupting pulmonary function and reducing mouse survival [69]. Irg1 deficiency is associated with greater amounts of IL- $1 \beta$ accumulation in airway of mice infected with laboratory strains of $P$. aeruginosa [59], confirming the anti-inflammatory and protective role this metabolite has in the setting of pulmonary infection. The abundance of both anti-inflammatory itaconate and pro-inflammatory succinate in the airway suggests that successful pathogens adapt to both macrophage metabolites in order to persist.

\section{$P$. aeruginosa induces and consumes airway itaconate}

In contrast to other organisms more susceptible to the electrophilic stress imposed by itaconate, such as Legionella pneumophila, S. aureus and Acinetobacter baumannii [70], P. aeruginosa has adapted to this metabolite, and are forced to degrade itaconate to survive. $P$. aeruginosa expresses 3 genes (ich, ict, icl) devoted to itaconate metabolism, enabling them to assimilate itaconate in the airway as carbon source [59, 70, 71] (Fig. 3a, b). This is a property shared by $M$. tuberculosis and the Aspergillus species [71, 72], suggesting an evolutive and conserved mechanism of adaption to host immunometabolites. The consumption of itaconate by the ict-ich-ccl locus generates acetyl-coA and pyruvate, which are feeders of the GS and TCA cycle, respectively and provide the components for biofilm production.

Clinical isolates of $P$. aeruginosa adapt to itaconate in vivo, with increased expression of the ict-ich-ccl locus, and induction of biofilm production. The clinical strains shift their preferred airway carbon source from succinate to itaconate. Itaconate stress causes increased bacterial outer membrane permeability, reducing the capacity of $P$. aeruginosa strains to transport LPS to their surface. These outer barrier function changes activate the algTalgD-MucA membrane stress repair system [73, 74], facilitating the replacement of LPS with more protective alginate polymers. Itaconate exposed laboratory strains of $P$. aeruginosa have diminished production of $\mathrm{O}$-side chains of LPS [59], confirming that these organisms divert carbohydrates from endotoxin synthesis to the generation of EPS, in response to membrane stress. These same findings were apparent in clinical isolates of $P$. aeruginosa from CF patients, which had been chronically exposed to airway itaconate. These CF strains had developed mutations in the pathways involved in LPS surface display, specifically in the lptD locus [59]. LptD is the LPS transporter embedded in the outer membrane that flips and anchors the endotoxin towards the extracellular side $[75,76]$. P. aeruginosa CF isolates from chronic infection fail to expose LPS nor do they induce macrophage release of succinate, as do environmental strains of $P$. aeruginosa. Of note, the EPS (alginate) produced by these isolates stimulates itaconate release from host immune cells [59]. The mechanisms by which $P$. aeruginosa EPS induces macrophage itaconate remains unclear. The changes in the display of EPS versus LPS on the surface of $P$. aeruginosa in chronic airway infection suggests that these organisms are forced to catabolize itaconate to prevent outer membrane disruption and biofilm clearance. The capacity of the ict-ich-ccl locus to provide $P$. aeruginosa with carbon structures such as acetyl-coA and pyruvate demonstrate its valuable contribution to the establishment of long-term lung colonization.

\section{Immune signaling activated by itaconate-adapted $P$. aeruginosa}

It is well appreciated that the induction of EPS moieties and biofilm production, especially the alginate overproducers characteristic of chronic infection in CF, correlate with the onset of intractable pulmonary infection [77]. Thus, the selection of these variants that induce itaconate production and then consume the metabolite, is of considerable clinical significance. The bacterial adaptive response, the production of EPS contributes to accumulation of phagocytes in the airways, but without resulting in bacterial clearance [78]. The prevailing dogma is that organisms enveloped in EPS, such as those with increased $\operatorname{alg} D$ or $p s l A$ resist phagocytosis and are "less" immunogenic $[24,79,80]$. However, when itaconateadapted clinical strains were examined for immunogenicity either in vivo or in vitro, they readily induced recruitment of immune cells into the airway [30, 59]. All of the strains stimulated variable amounts of IL- 6 and $\mathrm{TNF} \alpha$, and recruited greater numbers of neutrophils and monocytes than control bacteria [30,59]. The activation and release of substantial amounts of IL- $1 \beta$ was limited to the laboratory strain PAO1, perhaps due to its expression of LPS, flagella and toxins that directly activate the inflammasome and generate the release of succinate [39, 81] (Fig. 3c). The CF strains had lost expression of pathogen-associated molecular patterns (PAMPs) that are expected to induce IL-1 $\beta$, specially LPS, flagella and the type 3 toxins $[2,16,30,59,82]$, which was consistent with their failure to activate the release of succinate $[35,36]$ (Fig. 3d).

The immunogenicity of these $P$. aeruginosa CF isolates differed from that of the LPS-expressing laboratory strains, which have never been exposed to the itaconaterich airway before [30, 59] (Fig. 3d). Although these CF 
strains failed to induce IL-1 $\beta$, they stimulated myeloid cell reprograming generating itaconate. In sputum from CF patients infected with host-adapted $P$. aeruginosa, there is substantial accumulation of monocytes and macrophages that release itaconate. These findings indicate that the abundance of the immunometabolite itaconate has a major role in the selection of $P$. aeruginosa variants, promoting the display of EPS and not LPS on their surfaces and further contributing to the accumulation of itaconate and the establishment of intractable biofilms.

\section{Conclusions}

The availability of specific carbon sources in the airway, specifically, the relative amounts of succinate and itaconate are not typically considered major factors in susceptibility to infection. Instead, the relative resistance of bacteria to antibiotics, susceptibility to phagocytosis, and expression of toxins, either leukocidins or destructive proteases are typically considered the major factors promoting pulmonary infection [83-88]. However, it is important to consider how specific pathogens adapt to the airway and adjust their own metabolism to the biofilm mode of growth that promotes persistent infection. Historically, bacteria have been speciated according to their metabolic preferences, a methodology now supplanted by genomic studies, but remarkably useful in classifying specific organisms and their clinical relevance. Hence, $P$. aeruginosa has been classified as a "non-lactose fermenter" putting it in a selective category of opportunistic Gram-negative pathogens. As reviewed here, bacterial substrate preference turns out to be critical, first in the initial infection of the airway and then in the selection of proficient biofilm formers.

Both planktonic and biofilm associated bacteria persist over the course of infection, but at some point, the hostadapted strains predominate and an itaconate-dominant immuno-metabolic milieu is generated. Thus, the nature of the immunometabolites that are released during the course of $P$. aeruginosa infection and the multiple PAMPs that induce their excretion are a major factor in the success of this pathogen as a cause of chronic pulmonary infection. Further studies are needed to elucidate how $P$. aeruginosa and many other respiratory pathogens induce and exploit particular immunometabolic responses to cause acute and then transition into long-term disease. The characterization of these pathways would provide with new targets to control airway infections, such as identification of which metabolites released by host macrophage fuel toxin production, biofilms, development of antibiotic resistance and promotion of adaptive changes.

The ability of P.aeruginosa to exploit the production of immunometabolites that are produced as a component of host defense clearly adds to their success as pulmonary pathogens. Moreover, the infecting organisms display a range of metabolic activities, as well illustrated by the phenotypic heterogenicity of the $P$. aeruginosa isolates in the $\mathrm{CF}$ airway, ranging from mucoid $[15,78]$ to small colony variants [29], each of which exhibit distinct metabolic profiles. The efficacy of antimicrobial therapy against P.aeruginosa infection might be complemented by targeting some of these metabolic pathways that are activated in vivo, such as itaconate degradation by the ict-ich-ccl locus [59]. Inhibition of this protective response might render $P$. aeruginosa susceptible to itaconate toxicity, reducing bacterial loads and their ability to produce biofilm. In parallel, administration of compounds that block $P$. aeruginosa assimilation of succinate might prevent their adaptation and synthesis of EPS. By forcing the organisms to maintain a planktonic lifestyle, phagocytic clearance would be improved. Better understanding of the bacterial machinery, activated in vivo, that function to transport these metabolites would also be useful. Competitive inhibitors to selectively block bacterial uptake of succinate and itaconate from the environment would limit the ability of this pathogen to proliferate in the airway.

An alternative therapeutic approach could target host metabolism. Incorporation of exogenous PTEN into the CF mitochondria, which would reduce the accumulation of immunometabolites in the infected airway, is another approach that merits further investigation [30]. It is challenging to deliver this phosphatase inside airway cells in the CF lung, although its recombinant isoform PTEN-long can be successfully internalized into tumor cells due to its poly-cationic N-terminal domain [89]. As the currently available CFTR potentiator and corrector therapies, by increasing membrane associated CFTR, will also increase PTEN function [61], further suppressing the accumulation of succinate and itaconate may be very effective in limiting $P$. aeruginosa adaptation and persistence. The complex association between the airway immune-metabolic responses to infection and $P$. aeruginosa suggest that this approach might be successful.

\section{Abbreviations}

CFTR: Cystic fibrosis conductance transmembrane regulator; PTEN: Phosphatase and tensin homologue deleted on chromosome 10; ROS: Reactive oxygen species; IRG1: Immunosuppressive gene 1; SDH: Succinate dehydrogenase; NRF2: Nuclear factor-erythroid-2-related factor 2; HO-1: Hemeoxygenase 1; KEAP1: Kelch-like ECH-associated protein 1;TLR4:Toll-like receptor 4; LPS: Lipopolysaccharide; EPS: Extracellular polysaccharide; HIF1a: Hypoxida induced factor 1a; COPD: Chronic obstructive pulmonary disease; CF: Cystic fibrosis; NLRP3: Pyrin domain-containing protein 3; NADH: Nicotinamide adenine dinucleotide; IDH: Isocitrate dehydrogenase.

Acknowledgements

Not applicable. 


\section{Authors' contributions}

S.A.R. and A.P. analyzed the literature and wrote the manuscript. Both authors read and approved the final manuscript.

\section{Funding}

A.P. is supported by NIH 1R35HL135800; S.A.R. by a Vertex Research Innovation Award PG010094.

\section{Availability of data and materials}

This manuscript does not include data and material than can be shared.

\section{Ethics approval and consent to participate}

This manuscript does not include information that requires ethical approval or consents.

\section{Consent for publication}

We agree with the publication of this work.

\section{Competing interests}

Authors declare no conflicts of interest exist.

Received: 3 September 2020 Accepted: 30 November 2020

Published online: 10 December 2020

\section{References}

1. Riquelme SA, Ahn D, Prince A. Pseudomonas aeruginosa and Klebsiella pneumoniae adaptation to innate immune clearance mechanisms in the lung. J Innate Immun. 2018;10:442-54.

2. Riquelme SA, Wong Fok Lung T, Prince A. Pulmonary pathogens adapt to immune signaling metabolites in the airway. Front Immunol. 2020;11:385

3. Fernandez-Barat L, Ferrer M, De Rosa F, Gabarrus A, Esperatti M, Terraneo S, Rinaudo M, Li Bassi G, Torres A. Intensive care unit-acquired pneumonia due to Pseudomonas aeruginosa with and without multidrug resistance. J Infect. 2017;74:142-52.

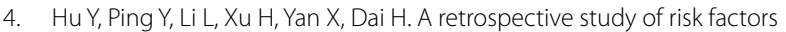
for carbapenem-resistant Klebsiella pneumoniae acquisition among ICU patients. J Infect Dev Ctries. 2016;10:208-13.

5. Bauer PR, Sampathkumar P. Methicillin-resistant Staphylococcus aureus infection in ICU: what is the best prevention strategy? Crit Care Med. 2017:45:1413-4.

6. Paling FP, Wolkewitz M, Bode LGM, Klein Klouwenberg PMC, Ong DSY, Depuydt P, de Bus L, Sifakis F, Bonten MJM, Kluytmans J. Staphylococcus aureus colonization at ICU admission as a risk factor for developing $S$. aureus ICU pneumonia. Clin Microbiol Infect. 2017:23:49 e49-49 e14.

7. Hendricks MR, Lashua LP, Fischer DK, Flitter BA, Eichinger KM, Durbin JE, Sarkar SN, Coyne CB, Empey KM, Bomberger JM. Respiratory syncytial virus infection enhances Pseudomonas aeruginosa biofilm growth through dysregulation of nutritional immunity. Proc Natl Acad Sci USA. 2016:113:1642-7.

8. Villeret B, Solhonne B, Straube M, Lemaire F, Cazes A, Garcia-Verdugo I, Sallenave JM. Influenza A virus pre-infection exacerbates Pseudomonas aeruginosa-mediated lung damage through increased MMP-9 expression, decreased elafin production and tissue resilience. Front Immunol. 2020;11:117.

9. Mulcahy ME, McLoughlin RM. Staphylococcus aureus and Influenza A virus: partners in coinfection. mBio. 2016;7(6):e02068-16.

10. Yu KO, Randolph AG, Agan AA, Yip WK, Truemper EJ, Weiss SL, Ackerman KG, Schwarz AJ, Giuliano JS Jr, Hall MW, et al. Staphylococcus aureus alpha-toxin response distinguishes respiratory virus-methicillin-resistant S. aureus coinfection in children. J Infect Dis. 2016;214:1638-46.

11. Garcia-Nunez M, Marti S, Puig C, Perez-Brocal V, Millares L, Santos S, Ardanuy C, Moya A, Linares J, Monso E. Bronchial microbiome, PA biofilmforming capacity and exacerbation in severe COPD patients colonized by P. aeruginosa. Future Microbiol. 2017;12:379-92.

12. Valderrey AD, Pozuelo MJ, Jimenez PA, Macia MD, Oliver A, Rotger R. Chronic colonization by Pseudomonas aeruginosa of patients with obstructive lung diseases: cystic fibrosis, bronchiectasis, and chronic obstructive pulmonary disease. Diagn Microbiol Infect Dis. 2010;68:20-7.
13. Ciofu O, Mandsberg LF, Bjarnsholt T, Wassermann T, Hoiby N. Genetic adaptation of Pseudomonas aeruginosa during chronic lung infection of patients with cystic fibrosis: strong and weak mutators with heterogeneous genetic backgrounds emerge in mucA and/or lasR mutants. Microbiology. 2010;156:1108-19.

14. Cohen TS, Prince A. Cystic fibrosis: a mucosal immunodeficiency syndrome. Nat Med. 2012;18:509-19.

15. Deretic V, Schurr MJ, Yu H. Pseudomonas aeruginosa, mucoidy and the chronic infection phenotype in cystic fibrosis. Trends Microbiol. 1995;3:351-6.

16. Winstanley C, O'Brien S, Brockhurst MA. Pseudomonas aeruginosa evolutionary adaptation and diversification in cystic fibrosis chronic lung infections. Trends Microbiol. 2016:24:327-37.

17. Archer NK, Mazaitis MJ, Costerton JW, Leid JG, Powers ME, Shirtliff ME. Staphylococcus aureus biofilms: properties, regulation, and roles in human disease. Virulence. 2011;2:445-59.

18. Ciofu O, Tolker-Nielsen T. Tolerance and resistance of Pseudomonas aeruginosa biofilms to antimicrobial agents — how P. aeruginosa can escape antibiotics. Front Microbiol. 2019;10:913.

19. O'Toole G, Kaplan HB, Kolter R. Biofilm formation as microbial development. Annu Rev Microbiol. 2000;54:49-79.

20. Lemire J, Alhasawi A, Appanna VP, Tharmalingam S, Appanna VD. Metabolic defence against oxidative stress: the road less travelled so far. J Appl Microbiol. 2017;123:798-809.

21. Boles BR, Singh PK. Endogenous oxidative stress produces diversity and adaptability in biofilm communities. Proc Natl Acad Sci USA. 2008;105:12503-8.

22. Chua SL, Ding Y, Liu Y, Cai Z, Zhou J, Swarup S, Drautz-Moses DI, Schuster SC, Kjelleberg S, Givskov M, Yang L. Reactive oxygen species drive evolution of pro-biofilm variants in pathogens by modulating cyclic-di-GMP levels. Open Biol. 2016:6:160162.

23. Franklin MJ, Nivens DE, Weadge JT, Howell PL. Biosynthesis of the Pseudomonas aeruginosa extracellular polysaccharides, alginate, Pel, and Psl. Front Microbiol. 2011;2:167.

24. Simpson JA, Smith SE, Dean RT. Scavenging by alginate of free radicals released by macrophages. Free Radic Biol Med. 1989;6:347-53.

25. Ahn S, Jung J, Jang IA, Madsen EL, Park W. Role of glyoxylate shunt in oxidative stress response. J Biol Chem. 2016;291:11928-38.

26. Dolan SK, Welch M. The glyoxylate shunt, 60 years on. Annu Rev Microbiol. 2018;72:309-30.

27. Maurice NM, Bedi B, Sadikot RT. Pseudomonas aeruginosa biofilms: host response and clinical implications in lung infections. Am J Respir Cell Mol Biol. 2018;58:428-39.

28. Lopez-Causape C, Sommer LM, Cabot G, Rubio R, Ocampo-Sosa AA, Johansen HK, Figuerola J, Canton R, Kidd TJ, Molin S, Oliver A. Evolution of the Pseudomonas aeruginosa mutational resistome in an international cystic fibrosis clone. Sci Rep. 2017;7:5555.

29. Starkey M, Hickman JH, Ma L, Zhang N, De Long S, Hinz A, Palacios S, Manoil C, Kirisits MJ, Starner TD, et al. Pseudomonas aeruginosa rugose small-colony variants have adaptations that likely promote persistence in the cystic fibrosis lung. J Bacteriol. 2009;191:3492-503.

30. Riquelme SA, Lozano C, Moustafa AM, Liimatta K, Tomlinson KL, Britto C, Khanal S, Gill SK, Narechania A, Azcona-Gutierrez JM, et al. CFTR-PTENdependent mitochondrial metabolic dysfunction promotes Pseudomonas aeruginosa airway infection. Sci Transl Med. 2019;11(499):eaav4634.

31. Elborn JS. Cystic fibrosis. Lancet. 2016;388:2519-31.

32. Quinn RA. Integrating microbiome and metabolome data to understand infectious airway disease. Am J Respir Crit Care Med. 2017;196:806-7.

33. Raghuvanshi R, Vasco K, Vazquez-Baeza Y, Jiang L, Morton JT, Li D, Gonzalez A, DeRight Goldasich L, Humphrey G, Ackermann G, et al. High-resolution longitudinal dynamics of the cystic fibrosis sputum microbiome and metabolome through antibiotic therapy. mSystems. 2020;5(3):e00292-20.

34. Quinn RA, Phelan WV, Whiteson KL, Garg N, Bailey BA, Lim YW, Conrad DJ, Dorrestein PC, Rohwer FL. Microbial, host and xenobiotic diversity in the cystic fibrosis sputum metabolome. ISME J. 2016;10:1483-98.

35. Tannahill GM, Curtis AM, Adamik J, Palsson-McDermott EM, McGettrick AF, Goel G, Frezza C, Bernard NJ, Kelly B, Foley NH, et al. Succinate is an inflammatory signal that induces IL-1beta through HIF-1alpha. Nature. 2013:496:238-42.

36. Corcoran SE, O'Neill LA. HIF1alpha and metabolic reprogramming in inflammation. J Clin Invest. 2016;126:3699-707. 
37. Mills EL, Kelly B, Logan A, Costa ASH, Varma M, Bryant CE, Tourlomousis P, Dabritz JHM, Gottlieb E, Latorre I, et al. Succinate dehydrogenase supports metabolic repurposing of mitochondria to drive inflammatory macrophages. Cell. 2016;167(457-470):e413.

38. Kelly B, O'Neill LA. Metabolic reprogramming in macrophages and dendritic cells in innate immunity. Cell Res. 2015;25:771-84.

39. Cohen TS, Prince AS. Activation of inflammasome signaling mediates pathology of acute $P$. aeruginosa pneumonia. J Clin Invest. 2013:123:1630-7.

40. Iannitti RG, Napolioni V, Oikonomou V, De Luca A, Galosi C, Pariano M, Massi-Benedetti C, Borghi M, Puccetti M, Lucidi V, et al. IL-1 receptor antagonist ameliorates inflammasome-dependent inflammation in murine and human cystic fibrosis. Nat Commun. 2016;7:10791.

41. Schultz MJ, Rijneveld AW, Florquin S, Edwards CK, Dinarello CA, van der Poll T. Role of interleukin-1 in the pulmonary immune response during Pseudomonas aeruginosa pneumonia. Am J Physiol Lung Cell Mol Physiol. 2002;282:L285-290

42. McElvaney OJ, Zaslona Z, Becker-Flegler K, Palsson-McDermott EM, Boland F, Gunaratnam C, Gulbins E, O'Neill LA, Reeves EP, McElvaney NG. Specific inhibition of the NLRP3 inflammasome as an antiinflammatory strategy in cystic fibrosis. Am J Respir Crit Care Med. 2019;200:1381-91.

43. Scambler T, Jarosz-Griffiths HH, Lara-Reyna S, Pathak S, Wong C, Holbrook J, Martinon F, Savic S, Peckham D, McDermott MF. ENaC-mediated sodium influx exacerbates NLRP3-dependent inflammation in cystic fibrosis. Elife. 2019;8:e49248.

44. Collier DN, Hager PW, Phibbs PV Jr. Catabolite repression control in the Pseudomonads. Res Microbiol. 1996;147:551-61.

45. Gorke B, Stulke J. Carbon catabolite repression in bacteria: many ways to make the most out of nutrients. Nat Rev Microbiol. 2008;6:613-24.

46. Rojo F. Carbon catabolite repression in Pseudomonas: optimizing metabolic versatility and interactions with the environment. FEMS Microbiol Rev. 2010;34:658-84.

47. Valentini $M$, Lapouge $K$. Catabolite repression in Pseudomonas aeruginosa PAO1 regulates the uptake of C 4 -dicarboxylates depending on succinate concentration. Environ Microbiol. 2013;15:1707-16.

48. O'Toole GA, Gibbs KA, Hager PW, Phibbs PV Jr, Kolter R. The global carbon metabolism regulator $\mathrm{Crc}$ is a component of a signal transduction pathway required for biofilm development by Pseudomonas aeruginosa. J Bacteriol. 2000;182:425-31.

49. Crousilles A, Dolan SK, Brear P, Chirgadze DY, Welch M. Gluconeogenic precursor availability regulates flux through the glyoxylate shunt in Pseudomonas aeruginosa. J Biol Chem. 2018;293:14260-9.

50. Flynn JM, Phan C, Hunter RC. Genome-wide survey of Pseudomonas aeruginosa PA14 reveals a role for the glyoxylate pathway and extracellular proteases in the utilization of mucin. Infect Immun. 2017;85(8):e00182-17.

51. Hagins JM, Scoffield J, Suh SJ, Silo-Suh L. Malate synthase expression is deregulated in the Pseudomonas aeruginosa cystic fibrosis isolate FRD1. Can J Microbiol. 2011;57:186-95.

52. Blouin MJ, Zhao Y, Zakikhani M, Algire C, Piura E, Pollak M. Loss of function of PTEN alters the relationship between glucose concentration and cell proliferation, increases glycolysis, and sensitizes cells to 2-deoxyglucose Cancer Lett. 2010;289:246-53.

53. Li Y, He L, Zeng N, Sahu D, Cadenas E, Shearn C, Li W, Stiles BL. Phosphatase and tensin homolog deleted on chromosome 10 (PTEN) signaling regulates mitochondrial biogenesis and respiration via estrogenrelated receptor alpha (ERRalpha). J Biol Chem. 2013;288:25007-24.

54. Liang H, He S, Yang J, Jia X, Wang P, Chen X, Zhang Z, Zou X, McNutt MA, Shen WH, Yin Y. PTENalpha, a PTEN isoform translated through alternative initiation, regulates mitochondrial function and energy metabolism. Cell Metab. 2014;19:836-48.

55. Naguib A, Mathew G, Reczek CR, Watrud K, Ambrico A, Herzka T, Salas IC, Lee MF, El-Amine N, Zheng W, et al. Mitochondrial complex I inhibitors expose a vulnerability for selective killing of Pten-null cells. Cell Rep. 2018;23:58-67.

56. Dominguez-Andres J, Novakovic B, LiY, Scicluna BP, Gresnigt MS, Arts RJW, Oosting M, Moorlag S, Groh LA, Zwaag J, et al. The itaconate pathway is a central regulatory node linking innate immune tolerance and trained immunity. Cell Metab. 2019;29(211-220):e215.

57. Murphy MP, O'Neill LAJ. Krebs cycle reimagined: the emerging roles of succinate and itaconate as signal transducers. Cell. 2018;174:780-4.
58. O'Neill LAJ, Artyomov MN. Itaconate: the poster child of metabolic reprogramming in macrophage function. Nat Rev Immunol. 2019;19(5):273-81.

59. Riquelme SA, Liimatta K, Wong Fok Lung T, Fields B, Ahn D, Chen D, Lozano C, Saenz Y, Uhlemann AC, Kahl BC, et al. Pseudomonas aeruginosa utilizes host-derived itaconate to redirect its metabolism to promote biofilm formation. Cell Metab. 2020;31:1091-1106 e1096.

60. Hobert JA, Mester JL, Moline J, Eng C. Elevated plasma succinate in PTEN, SDHB, and SDHD mutation-positive individuals. Genet Med. 2012;14:616-9.

61. Riquelme SA, Hopkins BD, Wolfe AL, DiMango E, Kitur K, Parsons R, Prince A. Cystic fibrosis transmembrane conductance regulator attaches tumor suppressor PTEN to the membrane and promotes anti Pseudomonas aeruginosa immunity. Immunity. 2017;47(1169-1181):e1167.

62. Zhang PX, Cheng J, Zou S, D'Souza AD, Koff JL, Lu J, Lee PJ, Krause DS, Egan ME, Bruscia EM. Pharmacological modulation of the AKT/microRNA199a-5p/CAV1 pathway ameliorates cystic fibrosis lung hyper-inflammation. Nat Commun. 2015;6:6221.

63. Lampropoulou V, Sergushichev A, Bambouskova M, Nair S, Vincent EE, Loginicheva E, Cervantes-Barragan L, Ma X, Huang SC, Griss T, et al. Itaconate links inhibition of succinate dehydrogenase with macrophage metabolic remodeling and regulation of inflammation. Cell Metab. 2016;24:158-66.

64. Mills EL, Ryan DG, Prag HA, Dikovskaya D, Menon D, Zaslona Z, Jedrychowski MP, Costa ASH, Higgins M, Hams E, et al. Itaconate is an anti-inflammatory metabolite that activates Nrf2 via alkylation of KEAP1. Nature. 2018;556:113-7.

65. Bambouskova M, Gorvel L, Lampropoulou V, Sergushichev A, Loginicheva E, Johnson K, Korenfeld D, Mathyer ME, Kim H, Huang LH, et al. Electrophilic properties of itaconate and derivatives regulate the IkappaBzetaATF3 inflammatory axis. Nature. 2018;556:501-4.

66. Hooftman A, Angiari S, Hester S, Corcoran SE, Runtsch MC, Ling C, Ruzek MC, Slivka PF, McGettrick AF, Banahan K, et al. The immunomodulatory metabolite itaconate modifies NLRP3 and inhibits inflammasome activation. Cell Metab. 2020;32(3):468-478.e7.

67. Cordes T, Wallace M, Michelucci A, Divakaruni AS, Sapcariu SC, Sousa C, Koseki H, Cabrales P, Murphy AN, Hiller K, Metallo CM. Immunoresponsive gene 1 and itaconate inhibit succinate dehydrogenase to modulate intracellular succinate levels. J Biol Chem. 2016;291:14274-84.

68. Qin W, Qin K, Zhang Y, Jia W, Chen Y, Cheng B, Peng L, Chen N, Liu Y, Zhou $W$, et al. S-Glycosylation-based cysteine profiling reveals regulation of glycolysis by itaconate. Nat Chem Biol. 2019;15:983-91.

69. Nair S, Huynh JP, Lampropoulou V, Loginicheva E, Esaulova E, Gounder AP, Boon ACM, Schwarzkopf EA, Bradstreet TR, Edelson BT, et al. Irg1 expression in myeloid cells prevents immunopathology during M. tuberculosis infection. J Exp Med. 2018;215:1035-45.

70. Naujoks J, Tabeling C, Dill BD, Hoffmann C, Brown AS, Kunze M, Kempa S, Peter A, Mollenkopf HJ, Dorhoi A, et al. IFNs modify the proteome of legionella-containing vacuoles and restrict infection via IRG1-derived itaconic acid. PLoS Pathog. 2016;12:e1005408.

71. Sasikaran J, Ziemski M, Zadora PK, Fleig A, Berg IA. Bacterial itaconate degradation promotes pathogenicity. Nat Chem Biol. 2014;10:371-7.

72. Wang H, Fedorov AA, Fedorov EV, Hunt DM, Rodgers A, Douglas HL, Garza-Garcia A, Bonanno JB, Almo SC, de Carvalho LPS. An essential bifunctional enzyme in Mycobacterium tuberculosis for itaconate dissimilation and leucine catabolism. Proc Natl Acad Sci USA. 2019;116:15907-13.

73. Lima S, Guo MS, Chaba R, Gross CA, Sauer RT. Dual molecular signals mediate the bacterial response to outer-membrane stress. Science. 2013:340:837-41.

74. Li S, Lou X, Xu Y, Teng X, Liu R, Zhang Q, Wu W, Wang Y, Bartlam M. Structural basis for the recognition of MucA by MucB and AlgU in Pseudomonas aeruginosa. FEBS J. 2019;286(24):4982-94.

75. Botos I, Majdalani N, Mayclin SJ, McCarthy JG, Lundquist K, Wojtowicz D, Barnard TJ, Gumbart JC, Buchanan SK. Structural and functional characterization of the LPS transporter LptDE from gram-negative pathogens. Structure. 2016;24:965-76.

76. Balibar CJ, Grabowicz M. Mutant alleles of IptD increase the permeability of Pseudomonas aeruginosa and define determinants of intrinsic resistance to antibiotics. Antimicrob Agents Chemother. 2016;60:845-54. 
77. Hoiby N, Ciofu O, Bjarnsholt T. Pseudomonas aeruginosa biofilms in cystic fibrosis. Future Microbiol. 2010;5:1663-74.

78. Malhotra S, Hayes D Jr, Wozniak DJ. Mucoid Pseudomonas aeruginosa and regional inflammation in the cystic fibrosis lung. J Cyst Fibros. 2019;18:796-803.

79. Mahenthiralingam E, Campbell ME, Speert DP. Nonmotility and phagocytic resistance of Pseudomonas aeruginosa isolates from chronically colonized patients with cystic fibrosis. Infect Immun. 1994;62:596-605

80. Mishra M, Byrd MS, Sergeant S, Azad AK, Parsek MR, McPhail L, Schlesinger LS, Wozniak DJ. Pseudomonas aeruginosa Psl polysaccharide reduces neutrophil phagocytosis and the oxidative response by limiting complement-mediated opsonization. Cell Microbiol. 2012;14:95-106.

81. Reyes Ruiz VM, Ramirez J, Naseer N, Palacio NM, Siddarthan IJ, Yan BM, Boyer MA, Pensinger DA, Sauer JD, Shin S. Broad detection of bacterial type III secretion system and flagellin proteins by the human NAIP/NLRC4 inflammasome. Proc Natl Acad Sci USA. 2017;114:13242-7.

82. Faure $E$, Kwong $K$, Nguyen D. Pseudomonas aeruginosa in chronic lung infections: how to adapt within the host? Front Immunol. 2018;9:2416.

83. Soong G, Parker D, Magargee M, Prince AS. The type III toxins of Pseudomonas aeruginosa disrupt epithelial barrier function. J Bacteriol. 2008; 190:2814-21.

84. Michalska M, Wolf P. Pseudomonas Exotoxin A: optimized by evolution for effective killing. Front Microbiol. 2015;6:963.
85. Kitur K, Parker D, Nieto P, Ahn DS, Cohen TS, Chung S, Wachtel S, Bueno S, Prince A. Toxin-induced necroptosis is a major mechanism of Staphylococcus aureus lung damage. PLoS Pathog. 2015;11:e1004820.

86. Bhattacharya M, Berends ETM, Zheng X, Hill PJ, Chan R, Torres VJ, Wozniak DJ. Leukocidins and the nuclease Nuc prevent neutrophil mediated killing of Staphylococcus aureus biofilms. Infect Immun. 2020;88(10):e00372-20.

87. Chan R, Buckley PT, O'Malley A, Sause WE, Alonzo F 3rd, Lubkin A, Boguslawski KM, Payne A, Fernandez J, Strohl WR, et al. Identification of biologic agents to neutralize the bicomponent leukocidins of Staphylococcus aureus. Sci Transl Med. 2019;11(475):eaat0882.

88. Spaan AN, van Strijp JAG, Torres VJ. Leukocidins: staphylococcal bicomponent pore-forming toxins find their receptors. Nat Rev Microbiol. 2017;15:435-47.

89. Hopkins BD, Fine B, Steinbach N, Dendy M, Rapp Z, Shaw J, Pappas K, Yu JS, Hodakoski C, Mense S, et al. A secreted PTEN phosphatase that enters cells to alter signaling and survival. Science. 2013;341:399-402.

\section{Publisher's Note}

Springer Nature remains neutral with regard to jurisdictional claims in published maps and institutional affiliations.
Ready to submit your research? Choose BMC and benefit from:

- fast, convenient online submission

- thorough peer review by experienced researchers in your field

- rapid publication on acceptance

- support for research data, including large and complex data types

- gold Open Access which fosters wider collaboration and increased citations

- maximum visibility for your research: over 100M website views per year

At BMC, research is always in progress.

Learn more biomedcentral.com/submissions 